\title{
Danza, creatividad y artefactos cognitivos ${ }^{1}$ \\ Dance, Creativity and Cognitive Artifacts
}

\author{
Jõ̃o QUEIROZ \\ Universidad Federal de Juiz de Fora \\ Daniella Aguiar \\ Universidad Federal de Uberlândia
}

\begin{abstract}
RESUMEN: La creatividad artística puede ser observada como una propiedad de la manipulación de artefactos cognitivos. Más específicamente, está distribuida a través de artefactos cognitivos, como oportunidad para el surgimiento de entidades y procesos sorprendentes. En danza, la manipulación de nuevos artefactos cognitivos, como técnicas de danza, softwares y notaciones, lleva a la transformación de "espacios conceptuales". Cuando se producen nuevos artefactos, o se usan oportunamente en nuevos contextos, observamos lo que Margaret Boden denomina "creatividad transformacional". Enfocamos nuestro trabajo en cómo la introducción y manipulación de nuevos artefactos en ejemplos históricos contribuye a la transformación de los espacios conceptuales de la danza.
\end{abstract}

ABSTRACT: Artistic creativity can be regarded as a property of cognitive artifacts manipulation. More specifically, creativity is distributed through cognitive artifacts, opening opportunities for new and surprising products and processes. In dance, the manipulation of new cognitive artifacts, such as dance techniques, software, notations, leads to the transformation of dance conceptual spaces. When new artifacts are designed, or used in new contexts, we observe, what Margaret Boden calls, "transformational creativity". Here, we focus on the introduction and manipulation of new cognitive artifacts in historical examples and explore how they contribute to the transformation of dance conceptual spaces.

PALABRAS CLAVE: danza, creatividad transformacional, artefactos cognitivos.

KEYWORDS: dance, transformational creativity, cognitive artifacts

RECIBIDO: 02 de junio de 2017 • ACEPTADO: 23 de enero de 2018

1 Traducción de Rosario Lázaro Igoa.

Interpretatio, 3.2, 2018-2019, pp. 207-224 



\section{Jỗo QUEIROZ}

Universidad Federal de Juiz de Fora

DANIELla AGUIAR

Universidad Federal de Uberlândia

\section{Danza, creatividad y artefactos cognitivos}

\section{Introducción}

Los humanos proyectan herramientas y artefactos cognitivos para modificar la percepción, la atención, la memoria, la categorización, y los diferentes modos de inferir y razonar. Como Andy Clark y muchos otros autores han defendido, somos constructores de nichos cognitivos que extienden la mente hacia el espacio para pensar mejor (Clark 1998, 2003). ¿Qué ocurre entonces con la creatividad artística? ¿De qué manera la creatividad artística depende de la exploración de herramientas para el pensamiento y de artefactos cognitivos?

En trabajos anteriores (Aguiar 2008; Aguiar \& Queiroz 2009, 2010), nos concentramos en la caracterización de las técnicas de danza como artefactos cognitivos, y en el constreñimiento ejercido por las técnicas en la acción de bailarines y coreógrafos. Entre las cuestiones que orientaron nuestra investigación, está la pregunta sobre cómo un entrenamiento influye, modula o restringe el aprendizaje y la creación artística de bailarines y coreógrafos. La cuestión abordada en esta ocasión está más enfocada en los efectos producidos por artefactos en espacios conceptuales y nichos cognitivos, cuando se relacionan con la creatividad. ¿Cómo constriñen los artefactos cognitivos a los procesos creativos en la danza? 
Creatividad es un término usado de modo poco sistemático, y muchas veces se basa en nociones vagas como 'talento', 'genialidad' o 'don' (Sternberg 1999). Para Margaret Boden, la creatividad es:

the ability to come up with ideas or artefacts that are new, surprising, and valuable. 'Ideas', here, includes concepts, poems, musical compositions, scientific theories, cooking recipes, choreography, jokes... and so on, and on. 'Artefacts' include paintings, sculpture, steam engines, vacuum cleaners, pottery, origami, penny whistles... and you can name many more (Boden 2012: 29). ${ }^{2}$

Según esta autora, hay tres tipos de creatividad: combinatoria, exploratoria y transformacional. El primero está relacionado con el surgimiento de nuevas ideas o artefactos a través de procesos combinatorios de ideas o artefactos ya conocidos. ${ }^{3}$

El segundo consiste en la exploración de espacios conceptuales, que son "estilos de pensamiento estructurado" (Boden 2012: 32). Los espacios conceptuales incluyen "ways of writing prose or poetry; styles of sculpture, painting, or music; theories in chemistry or biology; fashions of couture or choreography [...] in short, any disciplined way of thinking that's familiar to (and valued by) a certain social group" (Boden 2012: 32). ${ }^{4}$

Se trata de posibles "pensamientos" diversos en un determinado espacio conceptual. En la creatividad exploratoria, estas posibilidades son exploradas sin que haya modificación de la "estructura de los pensamientos".

2 "la habilidad de generar ideas o artefactos que son nuevos, sorprendentes y valiosos. "Ideas", aquí, incluye conceptos, poemas, composiciones musicales, teorías científicas, recetas, coreografías, bromas... y así sucesivamente. "Artefactos" incluye pinturas, esculturas, motores a vapor, aspiradoras, cerámica, origami, silbatos... y muchas cosas más". Todas las citas del inglés fueron traducidas por la traductora.

3 Es importante destacar que el término artefacto para Boden no corresponde a la noción de 'artefacto cognitivo' que usamos aquí. Para esa autora, artefacto es el producto material de un fenómeno creativo.

4 "modos de escribir prosa o poesía; estilos de escultura, pintura o música; teorías en química o biología; tendencias en alta costura o coreografía [...] resumiendo, cualquier modo disciplinado de pensamiento que es familiar a (y validado por) un determinado grupo social". 
El tercer tipo de creatividad "involves some transformation of one or more of the (relatively fundamental) dimensions defining the conceptual space concerned" (Boden 2012: 29). ${ }^{5}$

Un espacio conceptual se establece a través de:

a set of enabling constraints, which make possible the generation of structures lying within that space [...]. If one or more of these constraints is altered (or dropped), the space is transformed. Ideas that previously were impossible (relative to the original conceptual space) become conceivable. (Boden 1999: 352). ${ }^{6}$

En danza, podemos reconocer los espacios conceptuales por medio del conjunto de artefactos que constriñen la creación de nuevas entidades y procesos. Como ejemplos de lo anterior, citamos la técnica del ballet clásico, el uso de zapatillas de punta, la estructura coreográfica y la valorización del centro del palco escénico, artefactos que constriñen el espacio conceptual del ballet clásico. Cuando se introducen algunos artefactos (o se sustraen), el espacio conceptual se transforma. Por ejemplo, la eliminación de las zapatillas por Isadora Duncan y otras bailarinas de principios del siglo Xx hizo concebibles nuevas "ideas", inaugurando un nuevo espacio conceptual o nicho cognitivo. Como quedará claro en este trabajo, las nociones de espacio conceptual y de nicho cognitivo serán tratadas como análogas, como distintas perspectivas del mismo fenómeno para enfatizar diferentes propiedades.

A pesar de la centralidad, en la definición de Boden, de "ideas y artefactos", estos son considerados productos de la habilidad mental del sujeto o del agente creativo. Su abordaje es coherente con paradigmas computacionalistas en ciencia cognitiva, donde la cognición equivale al procesamiento de unidades internas, discretas y intencionales de información (Clark 1999). En estos paradigmas, el papel del contexto y de las herramientas externas es secundario (Zhang 1997;

\footnotetext{
5 "involucra alguna transformación de una o más de las dimensiones (relativamente fundamentales) que definen el espacio conceptual específico".

6 "un conjunto de constreñimientos habilitantes, que permiten la generación de estructuras dentro de ese espacio [...]. Si al menos uno de esos constreñimientos es alterado (o abandonado), el espacio se transforma. Las ideas que previamente eran imposibles (relativas al espacio conceptual original) se vuelven concebibles".
} 
Smith 1999). Opuesta a esta perspectiva, la Ciencia Cognitiva Situada (Gallagher 2009; Wheeler 2005; Clark \& Chalmers 1998; Clark 2010, 2006a, 2003, 1998; Chemero 2009) ha cuestionado la idea de que el cerebro y el cuerpo son criterios para la demarcación de las fronteras entre la cognición y el ambiente. Según este abordaje, la mente depende de herramientas no biológicas, y hay etapas decisivas de la cognición que ocurren fuera del cerebro/mente (Clark 2006b, 2010).

A partir de estas premisas, la creatividad deja de ser caracterizada como una "habilidad" de las mentes individuales para producir "ideas y artefactos", y se transforma en el resultado de la introducción y manipulación de artefactos cognitivos en espacios conceptuales o nichos cognitivos. Lo mismo ocurre a partir de la introducción y manipulación de artefactos cognitivos que ofrecen oportunidades para la transformación de espacios conceptuales o de nichos, por la exploración de lo que se encuentra disponible, o de lo que es proyectado para manipulación. Frecuentemente, se trata de entidades y procesos externos. Ofrecemos aquí algunos ejemplos de cómo la introducción y la manipulación de artefactos cognitivos han participado en la transformación y creación de nichos y espacios conceptuales en la danza.

\section{Artefactos cognitivos y creatividad}

De acuerdo con la Distributed Cognition Thesis (Tesis de la cognición distributiva), varias herramientas, tales como lápiz y papel, calculadoras, calendarios, mapas, notaciones, modelos, computadoras, listas de compras, señales de tránsito, unidades de medida, etc., se consideran artefactos no-biológicos que permiten operaciones cognitivas externas al cráneo (Norman 1993; Hutchins 1995). En este sentido, se llaman artefactos cognitivos. Su influencia en las capacidades cognitivas puede ser descrita como poseedora de una doble identidad. Por una parte, los artefactos cognitivos actúan sobre la eficiencia de resolución de problemas: pueden reducir el costo cognitivo de una operación (como cuando se usa una calculadora para realizar una división), aumentar su precisión y eficiencia (uso de una regla para medir un objeto en vez de adivinar sus dimensiones), o permitir nuevas capacidades que serían 
imposibles para el cerebro por sí mismo (uso de un diagrama gráfico para representar la relación simultánea entre un gran número de entidades e inferir sus patrones visuales específicos). Por otra parte, los artefactos cognitivos son también responsables de la existencia misma de ciertas tareas, es decir, participan en la creación de nuevos problemas y espacios-problema. El lenguaje, por ejemplo, es un artefacto cognitivo poderoso (Clark 2006a, 2006b), que enmarca y transforma los espacios-problema de tareas relacionadas con la memoria, la percepción, la navegación, las formas de generalización y categorización, los modos de inferencia, etcétera.

Los artefactos cognitivos tienen el poder de crear nuevos problemas, así como de crear medios para resolver estos problemas. Cuando se toman en cuenta estos dos aspectos, los artefactos cognitivos pueden ser vistos como formadores de la propia cognición: la dotan de necesidades y capacidades, y crean herramientas cada vez más especializadas para hacer frente a tareas cada vez más especializadas. La actividad cognitiva está altamente influenciada por el desarrollo histórico de los artefactos puestos a disposición de los agentes en determinados entornos.

Según Clark (2003: 3; 2006b), los seres humanos son "cíborg natos", porque nacen con la competencia de acoplar artefactos no biológicos para crear y resolver problemas complejos. Los artefactos cognitivos modifican y amplían las acciones y las tareas cognitivas, creando dominios de problemas y espacios de soluciones concebibles. Los artefactos crean espacios conceptuales, para usar la nomenclatura de Boden. Modifican las acciones en el ambiente, amplifican o intensifican habilidades, con la posibilidad de alterarlas drásticamente. Entre los ejemplos de los artefactos más mencionados, encontramos: lápiz y papel, notaciones, mapas, modelos, calendarios, ábacos, calculadoras, computadoras, internet, celulares, GPS, cuadernos de anotaciones, guarismos, brújulas, lenguajes.

Para Hutchins (1999: 126), los "artefactos cognitivos son objetos físicos hechos por humanos con el propósito de auxiliar, aumentar o mejorar la cognición". Pueden ayudar a la memoria, la clasificación y comparación de categorías, las inferencias analógicas, las formas de predicción y la anticipación de eventos. Norman (1993) sugiere que las estructuras mentales, además de objetos físicos, pueden ser considera- 
das artefactos cognitivos, tales como reglas, procedimientos, proverbios y fábulas. Así, podemos describir los artefactos cognitivos como entidades y procesos que producen efectos cognitivos (Hutchins 1999: 127). Estos construyen espacios, o nichos, especializados. La noción de que estamos inmersos en nichos más o menos estructurados de artefactos es una idea poco explorada todavía en las artes, y en la teoría y filosofía de la danza. Sus consecuencias más radicales indican que diversas actividades dependen de la manipulación de tipos específicos de artefactos, que proveen atajos (shortcuts) para la solución de problemas. De acuerdo con Hutchins:

Cognitive artifacts are always embedded in larger sociocultural systems that organize the practices in which they are used. The utility of a cognitive artifact depends on other processes that create the conditions and exploit the consequences of its use. In culturally elaborated activities, partial solutions to frequently encountered problems are often crystallized in practices, in knowledge, in material artifacts, and in social arrangements (Hutchins 1999: 127).?

Los artefactos son, por lo tanto, un fenómeno físico o culturalmente situado y distribuido en el tiempo y en el espacio. Si la creatividad es un fenómeno cognitivo, y si la cognición es situada y distribuida a través de artefactos, entonces la creatividad será un fenómeno distribuido y dependiente de artefactos.

En danza, desde una perspectiva computacionalista, el "genio creador" es un atributo de sujetos y agentes. Por ejemplo, en el ballet romántico y clásico, el libretista, frecuentemente escritor o músico, concibe los ballets, y el coreógrafo materializa sus ideas (Sasportes 1983). En otros momentos históricos, el coreógrafo se torna creador, haciendo del bailarín un mero ejecutor. Hoy en día, vemos diversas formas de relación entre coreógrafos y bailarines. En muchas de ellas, el bailarín es también considerado creador. Una de las consecuencias de nuestra

"los artefactos cognitivos están siempre incorporados a sistemas socioculturales más amplios que organizan las prácticas en las que son usados. La utilidad de un artefacto cognitivo depende de otros procesos que crean las condiciones y exploran las consecuencias de su uso. En actividades culturalmente elaboradas, las soluciones parciales a los problemas frecuentes se cristalizan normalmente en prácticas, en conocimiento, en artefactos materiales y en disposiciones sociales". 
argumentación es que desplaza el foco de la explicación sobre la creatividad del agente de la acción creativa, su locus, hacia un proceso causal, cuya distribución es difusa en el tiempo y en el espacio. El bailarín, coreógrafo o bailarín-coreógrafo, o, más específicamente, lo que ellos son capaces de concebir o hacer, es el resultado del acoplamiento ${ }^{8}$ de artefactos cognitivos en nichos cognitivos y espacios conceptuales.

\section{Creatividad y construcción de nicho}

La noción de nicho implica un ambiente y sus artefactos, pero no es reductible a ellos. En ecología, mientras que el ambiente indica el hábitat físico de un organismo, el nicho indica no solo su "dirección local", sino también su "profesión" (Odum 1959), su papel ecológico. En una definición más precisa, el nicho es un hipervolumen multidimensional cuyos ejes corresponden a los diversos factores ecológicos decisivos para la vida del organismo (Hoffmeyer 2008). Si extendemos el concepto a la cognición, la noción destaca la oferta de oportunidades (y límites) al pensamiento, en un proceso evolutivo. Un nicho cognitivo se define como conjuntos de espacios de problemas que demandan, y seleccionan, ciertas habilidades cognitivas. Una propiedad de los nichos es que son autoconstruidos. No hay factores ambientales previamente existentes a los cuales los organismos se adapten. Al contrario, los nichos coevolucionan con los organismos. La Niche Construction Theory (NCT) redefine nuestra comprensión de los procesos evolutivos (Laland \& O’brien 2011). En la evolución clásica darwiniana, el ambiente ejerce unilateralmente una presión selectiva sobre rasgos heredados. Dentro de la perspectiva de la NCT, los ambientes y organismos se influyen mutuamente. En el caso de los nichos cognitivos, sus construcciones están relacionadas con la coevolución de recursos, tales como los artefactos externos (Bickerton 2009).

En nuestra argumentación, la creatividad es una propiedad de la exploración de artefactos cognitivos y construcción de nichos. En el caso

Por el término acoplamiento nos referimos, en primer lugar, a su significado vernáculo de "acción o resultado de acoplar(-se), unir(-se) o ligar(-se) formando una unidad" (Dicionário Aulete Digital, traducción nuestra). 
específico que nos interesa aquí, los nichos representan modos establecidos de explorar artefactos cognitivos, incorporando oportunidades para la acción. Cuando se exploran tales oportunidades, de modo tal que se establecen nuevas (sorprendentes y valiosas) relaciones entre cognición y artefactos cognitivos (es decir, cuando el nicho cognitivo artístico es construido), observamos creatividad exploratoria. Cuando se introducen, o crean, nuevos artefactos y se modifican constreñimientos considerados fundamentales, se transforma el espacio conceptual y se crea otro nicho; y observamos entonces creatividad transformacional. Este proceso de construcción del nicho, que es asimismo la transformación de espacios conceptuales, ocurre a través de la exploración del desarrollo de nuevos artefactos cognitivos (en danza, por ejemplo, técnicas y equipamientos tales como zapatillas, palco escénico, notaciones, softwares).

\section{Danza, artefactos cognitivos y creación}

Los artefactos cognitivos constriñen la acción de los bailarines y coreógrafos en diferentes niveles de descripción. Podemos analizar el constreñimiento de los artefactos sobre la creación y el desarrollo de programas estéticos, o de paradigmas, y sobre la creación de trabajos de danza. Las técnicas codificadas de danza, los espacios de presentación, las ideas y conceptos sobre composición, y otros artefactos, construyen nichos y espacios culturales. La idea central aquí es que cuando se introducen nuevos artefactos se pueden construir nichos, inaugurando nuevos paradigmas, o nuevos espacios conceptuales. A continuación, ofrecemos ejemplos, y también indicaremos algunos de los principales artefactos que contribuyen en sus construcciones.

\section{Ballet clásico}

Podemos describir el ballet clásico como un sistema de constreñimientos distribuidos en varios niveles, que incluye sonidos y estructuras rítmicas, movimientos corporales, pares de cuerpos, vestuario, luces y espacio escénico. Tales elementos se constriñen entre sí. 
El ballet clásico corresponde a la construcción de un nicho, o de un espacio conceptual, que implicó la producción y la introducción de diversos artefactos cognitivos. La propia técnica del ballet se debe al desarrollo de un nuevo artefacto cognitivo, como ya hemos argumentado (Aguiar 2008; Aguiar y Queiroz 2009, 2010). Codificada en "pasos de danza", funciona como un artefacto para la creación coreográfica, basada en la sistematicidad combinatoria de signos discretos (pasos).

La construcción de este nicho está relacionada, entre diversos ejemplos, con el cambio en la posición relativa del observador, y tiene origen en la exploración renacentista de la perspectiva visual. Esta última es un artefacto cognitivo originado (o redescubierto) por la exploración renacentista de la perspectiva de punto único en las artes visuales. Para ser más precisos, por la introducción de esta herramienta en el Teatro Olímpico Vicenza, diseñado por Andrea Palladio al final del siglo XVI (Crabtree \& Beudert 2005). Antes del uso de teatros basados en la perspectiva de Leon Battista Alberti, las piezas de danza de la corte eran vistas en grandes salones, ya sea al mismo nivel que los bailarines, o en posiciones elevadas, en gradas. En este caso, se exploraban patrones geométricos de ocupación espacial de los bailarines.

\section{Artefacto cognitivo: perspectiva visual de punto único}

Podemos describir el espacio de performance como la materialización de las relaciones semióticas entre bailarín y audiencia. En la danza barroca, la audiencia estaba distribuida alrededor y encima del espacio de performance. La alteración del espacio de presentación hacia la ejecución en palcos escénicos italianos, elevados con respecto a la posición del espectador, tiene efectos decisivos en la evolución de la danza clásica (Mendes 1987: 28), lo que contribuye a morfologías de movimiento más verticalizadas. En el teatro de perspectiva de punto único, la audiencia está directamente enfrente al palco escénico y ve solo un lado de la escena. Este único lado es comúnmente denominado "cuarto muro invisible". El nuevo artefacto cognitivo transforma al cuarto muro en un lienzo - la danza pasa de ser un evento tridimensional a uno bidimensional-. 
Como un proceso acumulativo, la preferencia por la verticalidad, asociada a cambios temáticos en el ballet romántico, lleva a la introducción de otro artefacto decisivo: la zapatilla de punta (Monteiro 1999: 184185). Esta actúa en la construcción del nuevo nicho, transformando a la bailarina en un personaje etéreo. El en dehors, rotación externa de la articulación coxofemoral, y la técnica de la pirueta, fueron desarrolladas por Carlo Blasis como respuesta a la frontalidad de los palcos italianos (Monteiro 1999; Mendes 1987: 28). Asimismo, asociada a otros artefactos, la perspectiva contribuyó, por ejemplo, a la jerarquía en la ocupación espacial del palco que valora el centro y la relación entre los bailarines en escena.

Sumariamente, la perspectiva visual, combinada con otros artefactos, constriñe en la composición coreográfica las morfologías de movimiento y técnicas corporales, la relación jerárquica observada en el espacio, destacando el centro, y la relación de los primeros bailarines con el cuerpo de baile. La introducción de este artefacto en la concepción y construcción del espacio de presentación contribuye al establecimiento del espacio conceptual (o nicho), que es el ballet clásico. Esto genera una cascada de eventos que inaugura un nuevo conjunto de problemas en la danza teatral y un nuevo nicho cognitivo: el ballet clásico.

\section{Merce Cunningham}

Según Copeland (2004: 2), Cunningham revisó de forma radical diversos "principios fundamentales" de la danza: relación entre movimiento, música y ritmo; relación entre tiempo y percepción del espacio; desarrollo de una nueva técnica de danza; prácticas de composición basadas en "operaciones aleatorias"; entre otros. Para este autor, ningún coreógrafo enfrentó tan sistemáticamente tantas "premisas", creando caminos de desarrollo (Copeland 2004: 2). Tales "caminos" pueden ser descritos como el resultado de la introducción y manipulación de nuevos artefactos cognitivos: uso controlado de procedimientos basados en el azar; nueva concepción del espacio (espacio no jerárquico de relaciones); nueva concepción de la asociación entre música y danza (son 
independientes); nueva concepción de la morfología de movimientos corporales (independientes de la expresión emocional).

Las experiencias de Cunningham incluyen la exploración de artefactos para la producción de efectos aleatorios (manipulación de monedas, uso del I-Ching, o El libro de las mutaciones) como metodología para la creación coreográfica. La manipulación de monedas, en particular, puede ser considerada como un artefacto binario de protocomputación para crear nuevos espacios de problemas sintácticos. La exploración del azar tiene muchas consecuencias sobre varios aspectos de la composición, tales como el orden de secciones de la coreografía, el orden de la secuencia de movimientos, la duración, la dirección de los movimientos de los bailarines, la localización de estos en el espacio, la velocidad de realización de las secuencias, el orden de combinación de los pasos, el número de bailarines que aparecen en cada secuencia (Copeland 2004). Los artefactos usados son diversos: "rolling dice, picking cards, tossing coins, consulting the I Ching, numbering imperfections - specks, watermarks - on pieces of paper" (Copeland 2004: 62). ${ }^{9}$

El uso de operaciones aleatorias tiene diversos efectos sobre la historia de la creación en la danza. Las nuevas posibilidades de secuenciar movimientos producen una sintaxis inusual que constriñe a los bailarines a adquirir nuevas habilidades, y a reorganizar sus coordenadas motoras. De acuerdo con Copeland, "given the chance dictated nature of movement sequences in his work, it's not surprising that the ordering of phrases rarely seemed guided by a "natural" sense of flow (or even by anatomical logic)" (Copeland 2004: 32). ${ }^{10}$

Como en el ejemplo del ballet clásico, lo que observamos aquí es una cascada de eventos basados en la introducción de operaciones aleatorias, un nuevo artefacto cognitivo, que impacta en la danza en varios sentidos e inaugura un nuevo conjunto de problemas. Al sortear la ocupación del espacio por parte de los bailarines, la jerarquía es reestructurada; sin regiones privilegiadas en el espacio, el centro pasa a ser

\footnotetext{
9 "tirar los dados, elegir cartas, tirar monedas, consultar el I-Ching, enumerar imperfecciones - manchas, marcas de agua - en pedazos de papel".

10 "dada la naturaleza de las secuencias de movimiento dictadas por el azar en su trabajo, no es sorprendente que la ordenación de las frases raramente parezca guiada por un sentido 'natural' de flujo (o incluso por una lógica anatómica)".
} 
tan importante como cualquier región observable. Al asociar la relación entre la coreografía y la música al azar, estos niveles son concebidos nuevamente, de modo que se empieza a explorar un carácter "no-representacional". La danza se torna "movimiento del cuerpo", independiente de otros niveles de organización (música, vestuario, escenario). La composición, y sus relaciones con diversos niveles, es radicalmente alterada con la introducción de artefactos asociados al azar.

\section{Danza posmoderna americana}

La danza posmoderna americana, ${ }^{11}$ movimiento que comienza a principios de los sesentas en Nueva York, es otro ejemplo de la transformación del espacio conceptual, o construcción de nicho. Segun Banes, "The dances by the early post-modern choreographers were [...] urgent reconsiderations of the medium" (Banes 1987: XVII). ${ }^{12}$ Las principales modificaciones, con relación a la danza moderna americana y al trabajo de Cunningham, se pueden resumir así: (I) citas a la historia de la danza, en especial al ballet clásico y a la danza moderna; (II) nuevas formas de explorar la percepción del tiempo en la danza, como por ejemplo desarrollo de performances donde no hay clímax o relación entre principio, desarrollo o fin, donde el tiempo es "plano"; (III) diferentes usos del espacio, especialmente uso de nuevas áreas de presentación, como una galería de arte, una iglesia, un apartamento, y otros; (IV) nuevas formas de explorar vocabularios motores, introduciendo cuerpos sin entrenamiento y movimientos cotidianos "funcionales".

11 Según Banes (1987: xiv-xv), el término danza posmoderna puede acarrear algunas confusiones cuando es comparado con el término posmoderno usado en otras áreas, sobre todo por el hecho de que la danza moderna no fue completamente modernista. Muchas de las cuestiones exploradas por el arte moderno (artes visuales, teatro, arquitectura, etc.) son desarrolladas en la danza posmoderna. Para Banes, "danza moderna" y "danza posmoderna" no corresponden exactamente a lo que es considerado "moderno" y "posmoderno" en otras áreas. Así, Banes sugiere el uso del término danza posmoderna y restringe su uso a diversas características, para que no sea usado indiscriminadamente. Aquí seguimos su denominación.

12 "las danzas de los primeros coreógrafos posmodernos eran [...] reconsideraciones urgentes del medio [de la danza]”. 
La técnica de la danza, como se ha dicho, puede ser descrita como un artefacto cognitivo para crear en diversos paradigmas artísticos. En la danza posmoderna no se desarrolla ninguna técnica específica, y, al mismo tiempo, se utilizan diversas estrategias para la creación de vocabularios motores. Para Kirby, en la danza posmoderna:

the choreographer does not apply visual standards to the work. The view is an interior one: movement is not pre-selected for its characteristics but results from certain decisions, goals, plans, schemes, rules, concepts, or problems. Whatever actual movement occurs during the performance is acceptable as long as the limiting and controlling principles are adhered to (Kirby 1975: 3). ${ }^{13}$

La introducción de nuevos artefactos para la creación de movimientos, como reglas y juegos de improvisación, así como el uso de movimientos cotidianos, modifica radicalmente la relación entre técnica y estética en la historia de la danza (Aguiar 2008; Bales 2008). Las técnicas de danza dejan de ser el principal criterio para definir un paradigma de danza.

Simoni Forti, por ejemplo, presentó "Five dance constructions and some other things" (1961) [Cinco construcciones de danza y algunas otras cosas], en el loft de Yoko Ono, donde se introdujeron diversos artefactos - rampa, cajas, columpio e instrucciones verbales - (Banes 1987: 26). En este ejemplo, es notable cómo la introducción de diversos artefactos disponibles crea el vocabulario de movimientos. Forti también exploró la introducción y el uso de nuevos espacios de presentación. En ese trabajo, la estructura espacial, que distribuye en cinco espacios y cinco diferentes acontecimientos, modifica drásticamente la relación entre platea e intérpretes, que hasta las creaciones de Cunningham ocurría en un teatro separado física (espacio para platea y espacio para performance) y conceptualmente (cuarta pared).

13 "el coreógrafo no aplica patrones visuales al trabajo. La visión es interior: el movimiento no es preseleccionado por sus características [visuales], sino que resulta de ciertas decisiones, objetivos, planes, esquemas, reglas, conceptos, o problemas. Cualquier movimiento real que ocurra durante la performance es aceptable siempre que los principios de limitación y control sean respetados". 


\section{Discusión}

Desde nuestro punto de vista, la creatividad es un proceso centrado en el diseño y exploración de herramientas externas y de artefactos cognitivos. Los ejemplos aquí descritos ilustran cómo la introducción y manipulación de nuevos artefactos cognitivos contribuyó a la construcción de nichos cognitivos y a la transformación de espacios conceptuales, ejemplificando lo que Boden denomina "creatividad transformacional". El espacio, o el nicho, modificados por la introducción de artefactos, alteran premisas fundamentales de la danza. Muchas implicaciones derivan de este abordaje. Sabemos que la ciencia cognitiva tiene dificultades para lidiar con las dimensiones creativas de la cognición, particularmente bajo la influencia de paradigmas computacionalistas. Al mismo tiempo, la creatividad ha sido tratada como una experiencia irreductible por filósofos, antropólogos, investigadores en artes y por los propios artistas, en general, sufriendo por la falta de sistematicidad y referencias a nociones poco operacionales y misteriosas. La creatividad artística, en particular, ha sido con frecuencia considerada un fenómeno profundamente subjetivo y personal que se resiste a la explicación. En nuestro abordaje, la creatividad es causalmente distribuida en el tiempo y en el espacio. No se encuentra en una entidad (agente o persona creativa) o en una región o segmento temporal ("momento de insight"). El "artista creativo" se torna parte del proceso de construcción del nicho cognitivo, y transformación del espacio conceptual, y no a la inversa (es decir, el proceso creativo como inherente al artista). Algunas características individuales, tales como habilidades mentales o características psicológicas, pericia y entrenamiento, influyen en el proceso creativo artístico, en el sentido de que contribuyen a la exploración de oportunidades de construcción de nichos cognitivos.

Un enfoque usual e intuitivo observa la historia del arte como la historia de los artistas, redes de trabajo, y conjuntos de obras. Una consecuencia de nuestra perspectiva es que sugiere otro enfoque. Si la introducción de nuevos artefactos es inevitable en la historia creativa del arte, la historia del arte es la evolución de sistemas de varios niveles, orientada por el diseño de artefactos cognitivos nuevos. 


\section{Bibliografía}

AgUiAR, Daniella (2008). Sobre treinamentos técnicos de dança como coleções de artefatos cognitivos. Disertación (Maestría) - Curso de Dança, Universidade Federal da Bahia, Salvador. <https://repositorio.ufba.br/ri/handle/ ri/9046>, consultado por última vez el 12 de febrero de 2017.

Aguiar, Daniella y João QueIroz (2009). "Técnicas de dança \& artefatos cognitivos", en Cristiane Wosniak, Sandra Meyer y Sigrid Nora (eds.), Seminários de Dança: o que quer e o que pode (ess)a técnica? Joinville, Letradágua: 45-53.

Aguiar, Daniella y João QueIroz (2010). "Artefatos cognitivos e técnicas de dança”, Teccogs: Revista Digital de Tecnologias Cognitivas, núm. 4: 49-59. <http://www4.pucsp.br/pos/tidd/teccogs/artigos/2010/edicao_4/4artefatos_cognitivos_e_tecnica_de_danca-daniella_aguiar-joao_queiroz. pdf $>$, consultado por última vez el 26 de abril de 2016.

BALES, Melanie (2008). "Training as the medium through which", en Melanie Bales y Rebecca Nettl-Fiol (eds.), Eclectic Bodies: Evolving practices in dance training. Urbana, University of Illinois Press: 28-42.

Banes, Sally (1987). Terpsichore in Sneakers: Post-Modern Dance. Middletown, Wesleyan.

Boden, Margaret A. (1999). "Computer Models of Creativity", en Robert J. Sternberg (ed.), Handbook of Creativity. Nueva York, Cambridge University Press: 351-372.

Boden, Margaret A. (2012). Creativity and Art: Three Roads to Surprise. Oxford, Oxford University Press.

Bickerton, Derek (2009). Adam's Tongue. Nueva York, Hill and Wang.

CHEMERo, Anthony (2009). Radical Embodied Cognitive Science. Massachusetts, The MIT Press.

Clark, Andy (1998). Being There: Putting Brain, Body, and World Together Again. Massachusetts, A Bradford Book.

Clark, Andy (1999). “An embodied cognitive science?", Trends in Cognitive Sciences, Cambridge, núm. 3: 345-351.

Clark, Andy (2003). Natural born-cyborgs: Minds, Technologies, and the Future of Human Intelligence. Oxford, Oxford University Press.

Clark, Andy (2006a). "Memento's Revenge: The Extended Mind, Extended", en Richard Menary (ed.), Objections and Replies to the Extended Mind. Aldershot, Ashgate: 1-43.

Clark, Andy (2006b). "Language, embodiment, and the cognitive niche", Trends in Cognitive Sciences, Cambridge, vol. 10, núm. 8: 370-374.

Clark, Andy (2010). Supersizing the Mind: Embodiment, Action, and Cognitive Extension. Oxford, Oxford University Press. 
Clark, Andy y David Chalmers (1998). "The Extended Mind”, Analysis, [s.p.i.], enero, vol. 58, núm. 1: 7-19.

Copeland, Roger (2004). Merce Cunningham: The Modernizing of Modern Dance. Nueva York, Routledge.

Dicionário Aulete Digital. <http://www.auletedigital.com.br/>, consultado por última vez el 27 de febrero de 2008.

GallaGHER, Shaun (2009). "Philosophical antecedents of situated cognition", en Philip Robins y Murat Aydede (eds.), The Cambridge Handbook of Situated Cognition. Cambridge, Cambridge University Press: 35-54.

HOFFMEYER, Jesper (2008). “The Semiotic Niche”, Journal of Mediterranean Ecology, Reggio Emilia, vol. 9: 5-30.

Hutchins, Edwin (1999). "Cognitive artifacts", en Robert A. Wilson y Frank C. Keil (eds.), The MIT Encyclopedia of the Cognitive Sciences. Massachusetts, The MIT Press: 126-127.

KIRBY, Michael (1975). "Introduction". The Drama Review, [s.p.i.], vol. 19, núm. 65.

LALAND, Kevin N. y Michael J. O'BRIEN (2011). “Cultural Niche Construction: An Introduction”, Biological Theory, [s.p.i.], núm. 6: 191-202.

Mendes GarCia, Miriam (1987). A dança. São Paulo, Editora Ática.

Monteiro, Mariana (1999). "Balé, tradição e ruptura", en Roberto Pereira y Silvia Soter (eds.), Lições de dança 1. Río de Janeiro, UniverCidade Editora: $169-189$.

Norman, Donald A. (1993). Things That Make Us Smart. Reading, MA, Addison Wesley.

Odum, E. (1959). Fundamentals of Ecology. Filadelfia, W. B. Saunders.

SASPORTES, José (1983). Pensar a dança: a reflexão estética de Mallarmé a Cocteau. Vila da Maia, Imprensa Nacional-Casa da moeda.

Sмiтн, Brian Cantwell (1999). "Situatedness/Embeddedness", en Robert A. Wilson y Frank C. Keil (eds.), The MIT Encyclopedia of the Cognitive Sciences. Massachusetts, The MIT Press: 769-770.

Sternberg, Robert J (1999). Handbook of Creativity. Cambridge, Cambridge University Press.

WheELER, Michael (2005). Reconstructing the Cognitive World: The Next Step. Massachusetts, The MIT Press.

Zhang, Jiajie (1997). "The Nature of External Representations in Problem Solving”, Cognitive Science, [s.p.i.], vol. 21, núm. 2: 179-217. 